\title{
Improving Safety in Laparoscopic Cholecystectomy: Reply
}

\author{
Marissa Adriana Johanna Nijssen ${ }^{1} \cdot J^{\prime}$ ennifer Schreinemakers ${ }^{1} \cdot$ Zainna Meyer $^{1} \cdot$ \\ George van der Schelling ${ }^{1} \cdot$ Rogier Crolla ${ }^{1} \cdot$ Arjen Rijken $^{1}$
}

Published online: 25 November 2015

(C) Société Internationale de Chirurgie 2015

To the Editor,

Thank you for the response to our article [1], we have read the letter to the editor with interest. As the authors, we are convinced that a culture of safety in performing laparoscopic cholecystectomy as in any other procedure is indeed necessary. We concur with the suggested four steps by the authors to ensure a safe laparoscopic cholecystectomy [2]. Following these steps not only ensure safety but also will often result in achieving CVS. After reviewing the videos of the laparoscopic cholecystectomies, we felt that in many cases, CVS could have been reached when in fact it was not. It is surprising that although surgeons believe they have reached a critical view of safety (CVS), they actually have not. This is a potentially harmful situation.

The Dutch guidelines [3] not only recommend attaining CVS, but actually recommend a stop point to evaluate CVS by taking a picture as well. It is questionable whether or not this is common practice.

In case CVS cannot be reached safely, we agree with the suggested alternatives by Connor et al. Training to become aware of these alternatives remains important. In our opinion, not only spatial awareness, but also continuing education after surgical training is a requirement. The change in behaviour and critical self-reflection of knowledge, technical and procedural skills are important to improve safety in this and other surgical procedures.

\section{References}

1. Nijssen MAJ, Schreinmakers JMJ, Meyer Z, van der Schelling GP, Crolla RMPH, Rijken AM (2015) Complications after laparoscopic cholecystectomy: a video evaluation study of whether the critical view of safety was reached. World J Surg 39:1798-1803. doi:10.1007/s00268-015-2993-9

2. Connor SJ, Perry W, Nathanson L, Hugh TJ, Hugh TB (2014) Using a standardised method for laparoscopic cholecystectomy to create a concept operation-specific checklist. HPB 16(5):422-429

3. Dutch guideline on gallstones. http://www.heelkunde.nl/uploads/_ 6/re/_6reZZkgrYUAuCG6uvcN-A/richtlijn_galsteen.pdf. Accessed 21 Sept 2015
Marissa Adriana Johanna Nijssen mnijssen@amphia.nl

1 Department of Surgery, Amphia Hospital, Breda, The Netherlands 